\section{Knee Loading Stimulates Bone Formation in Tail-Suspended Mouse Hindlimb}

\author{
Liming Zhao', Todd Dodge ${ }^{1}$, Eric Ledet ${ }^{2}$, and \\ Hiroki Yokota, ${ }^{1,3 *}$ \\ Department of Biomedical Engineering, \\ ${ }^{1}$ Indiana University - Purdue University Indianapolis, \\ Indianapolis, IN 46202, USA \\ ${ }^{2}$ Department of Biomedical Engineering, Rensselaer \\ Polytechnic Institute, Troy, NY 12180, USA \\ ${ }^{3}$ Department of Anatomy and Cell Biology, Indiana \\ University School of Medicine, Indianapolis, IN \\ 46202, USA
}

\section{Abstract}

Bone is a dynamic tissue that is constantly remodeling. However, for individuals requiring bed rest or astronauts exposed to microgravity in space, a lack of normal loaded activities leads to disuse osteoporosis. We examined whether knee loading increases bone mineral density (BMD) of both control and tailsuspended hindlimbs. We also examined whether the phosphorylation levels of Akt in phosphoinositide 3-kinase (PI3K) signaling and eukaryotic initiation factor $2 a$ (elF2a) in an integrated stress response (ISR) is altered in the loaded femur and tibia. Knee loading was applied to the right hindlimb using a custommade piezoelectric mechanical loader. The BMD of control and tail-suspended hindlimbs was determined by PIXImus imaging, and Western blot analysis was performed to determine the phosphorylation levels of Akt and elF2a. The results revealed that knee loading significantly increased the BMD in the loaded limb of tailsuspended mice. Compared to non-loaded limbs in the hindlimb-suspended mice, loaded limbs demonstrated a $6.6 \%$ increase in BMD. In the control group, the loaded limb showed a $7.4 \%$ increase in BMD over the non-loaded limb. Furthermore, knee loading activated PI3K signaling through the induction of Akt phosphorylation and reduced ISR by the suppression of elF2a phosphorylation. The present study thus supports knee loading as a potential loading method of preventing the detrimental effects of disuse due to unloading. (C)2011 Jpn. Soc. Biol. Sci. Space; Article ID: 112502010

Received: July 23, 2011; Accepted: Aug. 10, 2011

*To whom correspondence should be addressed:

Tel.:+1-317-278-5177 Fax: +1-317-278-2455;

E-mail: hyokota@iupui.edu
Keywords: unloading, knee loading, bone mineral density, elF2a

\section{Introduction}

Bone is a constantly adapting tissue that continually undergoes remodeling to repair damage and strengthen regions that are exposed to loads. Normal physical activities, such as walking, running, and jumping increase the strength of bone (Bergmann et al., 2010; Qin and Lam, 2009). These everyday activities and exercises, however, cannot be performed by individuals on bed rest, especially elderly patients. In addition, astronauts in space have limited exposure to mechanical loads. When exposed to these limited-load conditions, individuals may experience disuse osteoporosis in normally-loaded bones, such as the femur and tibia (Akhter et al., 2011; Sievänen, 2010). For instance, the bone mineral density (BMD) of the lower limbs of astronauts can decrease by up to $1.6 \%$ each month in microgravity (Rubin et al., 2001). The addition of growth hormones, dietary calcium intake, and muscle stimulation have been used in attempts to reverse bone loss, but have seen limited success (MoreyHolton and Globus, 1998). It is thus useful to develop a mechanical loading method that can easily be used by elderly individuals and astronauts in space.

Various loading modalities such as whole-body vibration, axial loading, and bending have been examined to stimulate bone formation (Ozcivici et al., 2010; Zhang et al., 2006). Joint loading is a recently developed modality that applies moderate lateral loads to the metaphysis of a synovial joint (Zhang et al., 2009). Knee loading is reported to induce bone formation throughout the length of the femur and tibia. Elbow loading only requires approximately one-quarter of the force required to stimulate bone formation using the axial-loading modality (Zhang et al., 2006). In this study, unloading was simulated by tail suspension and anabolic effects of knee loading during a 5-day tail-suspension period were evaluated.

To evaluate efficacy of knee loading for unloaded long bones, two primary questions were addressed: Does knee loading enhance bone formation in an unloaded limb? If yes, is its anabolic effect linked to the activation of phosphoinositide 3-kinase (PI3K) signaling and the suppression of an integrated stress response (ISR)? We hypothesized that knee loading increases BMD of unloaded long bones, and that it elevates the phosphorylation of Akt in the PI3K pathway and reduces ISR through suppression of the phosphorylation of eukaryotic initiation factor 2 subunit alpha (elF2a). Akt is a signaling molecule in $\mathrm{PI} 3 \mathrm{~K}$, which is involves cellular growth and proliferation (Zhang et al., 2009; Cantley, 2002). During a stress response induced by various stress inducers such as radiation, hypoxia, and nutrient deprivation, phosphorylation of elF2a leads to the suppression of translational machinery and apoptosis (Hirasawa et al., 2010; Proud, 2005; Ron et al., 2007). To test the hypotheses, we employed a mouse tailsuspension model (Morey-Holton and Globus, 1998; Iwamoto et al., 2005). 


\section{Materials and Methods}

\section{Animals}

All procedures performed in this study were in accordance with the Indiana University Animal Care and Use Committee Guidelines. We employed 23 female C57BL/6 wildtype mice ( 12 weeks of age; Harlan Sprague-Dawley, Inc.), in which 12 mice were used for the unloaded (hindlimb-suspended) group and 11 mice used for the control group. The animals were allowed to acclimate for 1 week before the experiment.

\section{Hindlimb suspension}

Mice were anesthetized by inhalation of isoflurane, and the tail was inserted into a plastic tube of the tail harness. The animal was placed head-down at approximately a $30-40^{\circ}$ angle that prohibited the hindlimbs from reaching the ground (Fig. 1A). The tail harness was attached to a metal swivel (fishing wire) and connected to a metal support that horizontally spanned a housing cage from one corner to the other. The mouse was able to move along the horizontal metal support and access about $80 \%$ of the cage. Water (hydrogel placed on floor) and chow (placed on floor) were provided ad libitum in a plastic dish throughout the experiment.

\section{Knee loading}

Knee loading was applied to the right knee of the control and tail-suspended mice. The mouse was placed in an anesthetic induction chamber to cause sedation and then mask-anesthetized using $2.0 \%$ isoflurane. Knee loading was conducted with a custom-made piezoelectric mechanical loader (Zhang et al., 2006; Fig. 1A). The loading was applied in the lateral-medial direction with 1 $\mathrm{N}$ force (peak-to-peak) at $5 \mathrm{~Hz}$ for $5 \mathrm{~min}$. The left knee was used as a sham loading control, where the knee was placed under the loading rod for $5 \mathrm{~min}$ in the same procedure used for the right knee without applying a voltage signal to the loader. During the procedure tail suspension was temporarily released. However, mice in the tail-suspension group did not receive regular gravity force in their hindlimb since they were anesthetized and laying down. The loading procedure was repeated once each day for 5 days. After loading, the mouse was allowed normal cage activities.

\section{Western blotting}

On day 5, animals were euthanized and the tibiae and femora were harvested $1 \mathrm{~h}$ after knee loading. Soft surrounding tissues were dissected out from the samples, which were then ground with a mortar and pestle in a RIPA lysis buffer containing inhibitors for proteases and phosphatases (Calbiochem). Isolated proteins were fractionated using $10 \%$ SDS gels and electro-transferred to Immobilon-P membranes (Millipore). Immunoblots were carried out using antibodies specific to Akt, phospho-Akt, elF2a (Cell Signaling); phospho-elF2a (pS52) (Invitorgen);
A
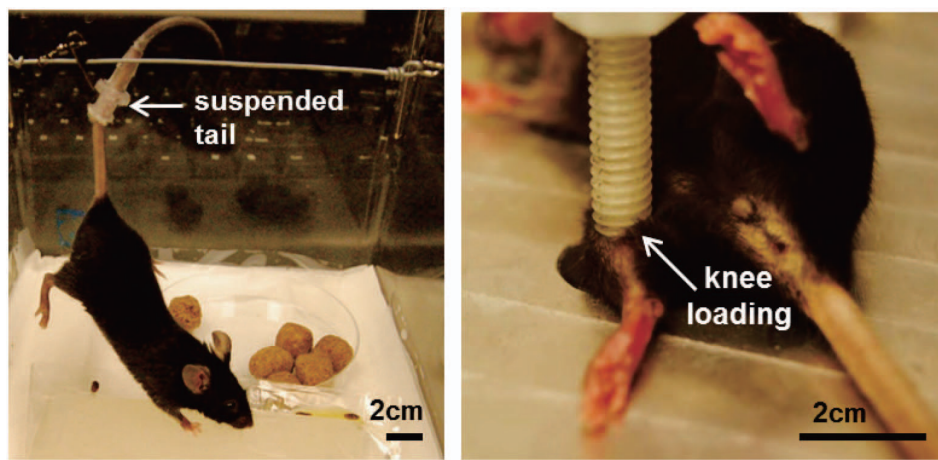

B

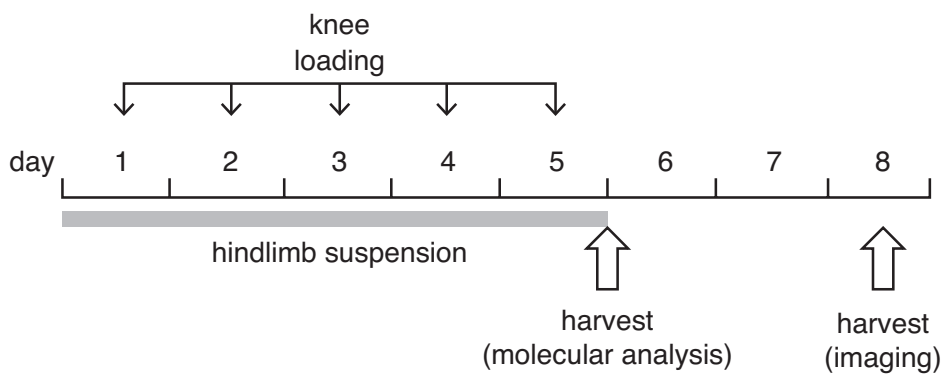

Fig. 1. Experimental setup. (A) Hindlimb suspension model (left) and knee loading modality (right). Scale bar $=2 \mathrm{~cm}$. (B) Experimental timeline. Tail suspension was applied for the first 5 days, and knee loading was conducted 5 min/day for 5 consecutive days. One hour after knee loading on day 5 , animals were harvested and Western blot analysis was performed. On day 8, additional animals were harvested and PIXImus imaging was conducted. Note that the described timeline was selected since PI3K signaling in the protein analysis is induced by mechanical loading immediately on the loading day but the alteration in BMD is an outcome of bone formation/remodeling that takes at least a few days after the loading bout. 
and $\beta$-actin (Sigma). After incubation with anti-rabbit IgG (Cell Signaling) or anti-mouse IgG (Amersham) antibodies conjugated with HRP, signals were detected with ECL chemiluminescence. Images were captured using a Fujifilm Luminescent image analyzer and analyzed using Adobe Photoshop (version 7.0).

\section{Determination of $B M D$}

On day 8 , mice were anesthetized in an anesthetic induction chamber and mask-anesthetized using 2.0\% isoflurane. A dual-energy $\mathrm{x}$-ray absorpiometry (Lunar PIXImus 2) machine (GE Medical Systems, Madison, WI), equipped with the Lunar PIXImus 2 software version 2.0, was used to scan the entire body of the mouse. After completion of the scan of the whole body, region of interest boxes were drawn around each limb and vertebrae L $1-L 6$. Bone mineral density $\left(\mathrm{g} / \mathrm{cm}^{2}\right)$ measurements were obtained from the PIXImus software.

\section{Statistical analysis}

The mean and standard deviation of all data was calculated, and 2-way ANOVA was conducted to evaluate

A

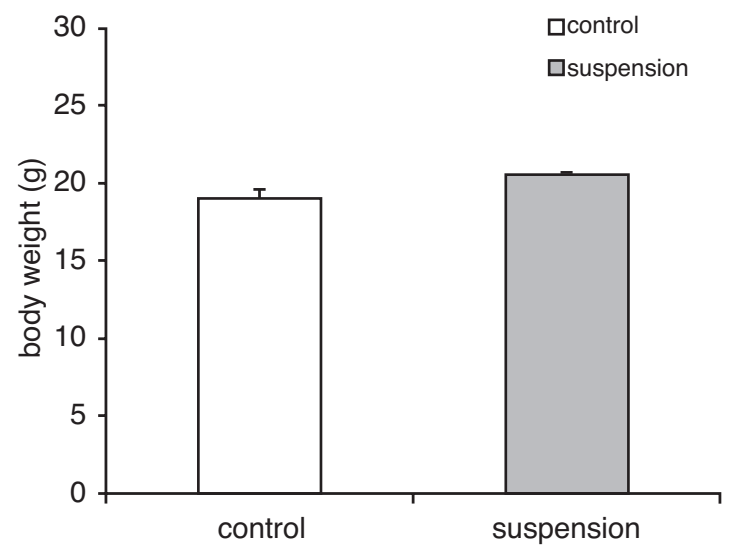

B

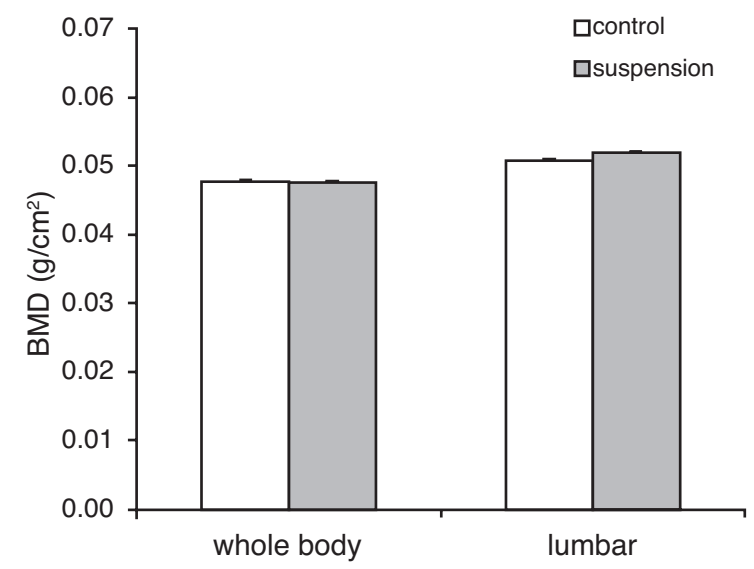

Fig. 2. Overall animal assessments on day 8. (A) Body weight. (B) BMD of whole body and lumbers. statistical significance for cross-comparisons between control vs. suspension, and knee loading vs. non-knee loading. P-values of less than $0.05\left(^{*}\right)$ were determined to be significant, with $p$-values of less than $\left.0.01{ }^{(*}\right)$ and $\left.0.0011^{* * *}\right)$ denoting higher levels of significance.

\section{Results}

\section{Body weight and bone mineral density measurements}

Upon completion of the hindlimb suspension tests on day 5 , animal body weight and bone mineral density were measured. The mean weights of the control and hindlimb-suspended mice were $20.63 \pm 0.13 \mathrm{~g}$ and 18.97 $\pm 0.58 \mathrm{~g}$, respectively, and no statistical difference was observed between the two groups (Fig. 2A). The BMD of the whole body and lumbar sections of the animals was measured using PIXImus imaging. The average BMD of the whole body of control animals was $0.0478 \pm 0.0002 \mathrm{~g} /$ $\mathrm{cm}^{2}$, while the lumbar section exhibited a BMD of 0.0508 $\pm 0.0003 \mathrm{~g} / \mathrm{cm}^{2}$. In suspended animals, the average BMD

A
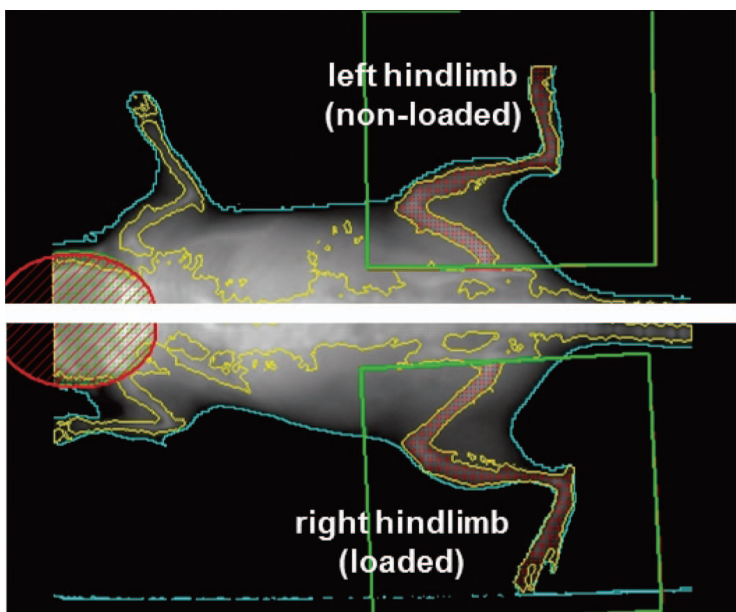

B

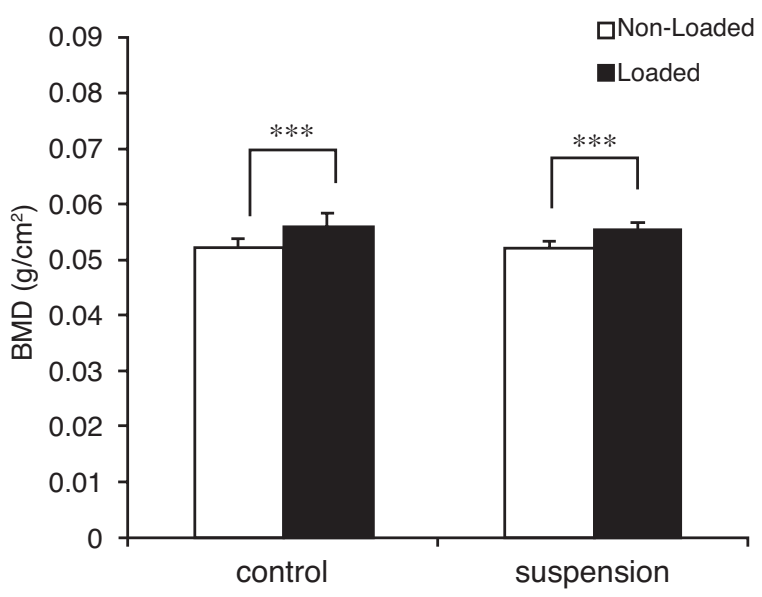

Fig. 3. PIXImus measurements of hindlimbs on day 8. (A) Region of interests for the left hindlimb (non-loaded) and the right hindlimb (loaded). (B) BMD measurements of the loaded and non-loaded hindlimbs for the tail-suspended and control animals. 
of the whole body was $0.0477 \pm 0.0001 \mathrm{~g} / \mathrm{cm}^{2}$ and the lumbar section was $0.0519 \pm 0.0002 \mathrm{~g} / \mathrm{cm}^{2}$. While the mean lumbar BMD was slightly higher than the average whole body BMD, the BMD in each region for both the control and hindlimb-suspended mice was not statistically different (Fig. 2B).

\section{Increased BMD in loaded limbs of control and hindlimb-suspended mice}

On day 8, PIXImus imaging was used to determine the BMD of the loaded (right) and non-loaded (left) hindlimbs (Fig. 3A). The BMD of loaded and non-loaded control limbs was found to be $0.0562 \pm 0.0021 \mathrm{~g} / \mathrm{cm}^{2}$ and $0.0524 \pm 0.0015 \mathrm{~g} / \mathrm{cm}^{2}$, respectively, an increase of $7.4 \%$ in loaded limbs. In the suspended group, the BMD of loaded and non-loaded limbs was $0.0556 \pm 0.0012 \mathrm{~g} /$ $\mathrm{cm}^{2}$ and $0.0521 \pm 0.0012 \mathrm{~g} / \mathrm{cm}^{2}$, respectively, an increase

A

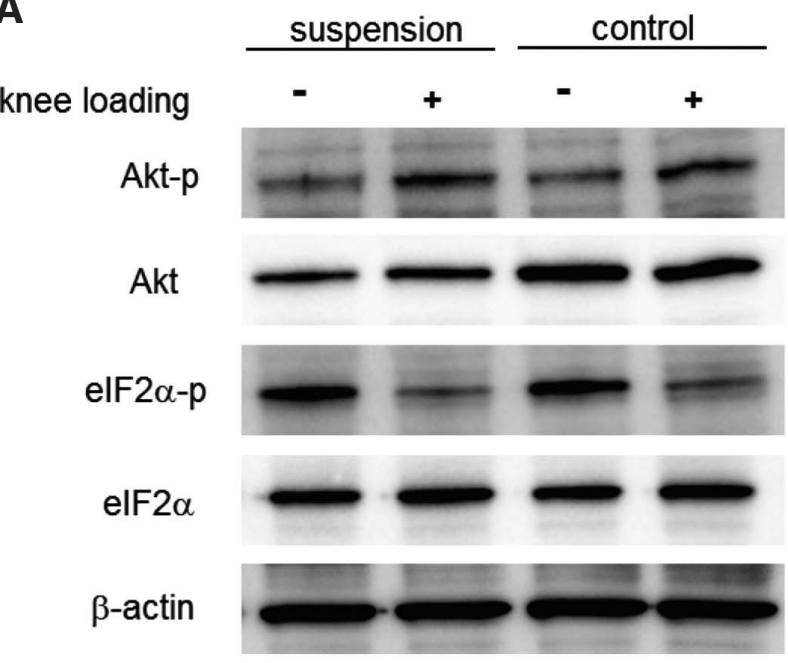

B

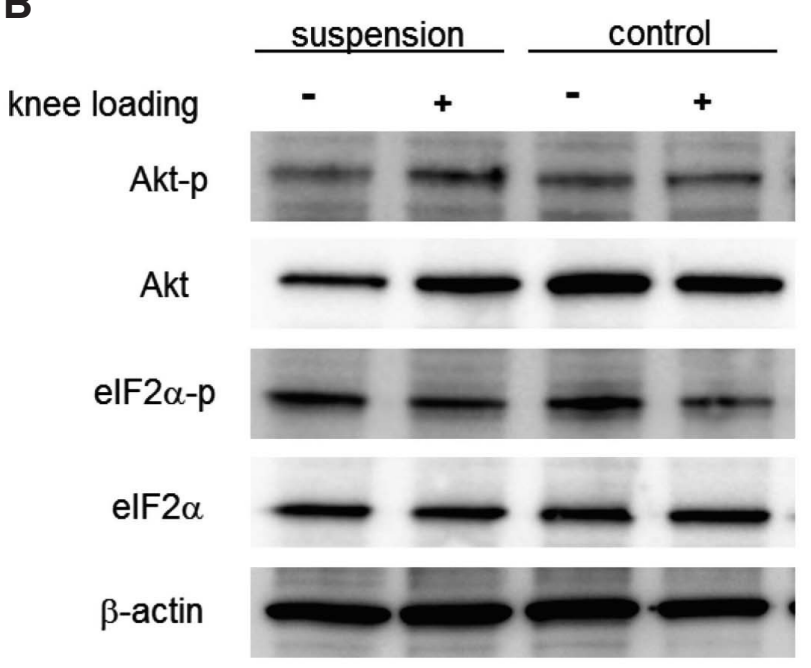

Fig. 4. Western blot analysis for elF2a, elF2a-p, Akt, and Akt-p. Femur and tibia samples were harvested $1 \mathrm{~h}$ after the last loading on day 5. (A) Femur samples. (B) Tibia samples. of $6.6 \%$ in loaded limbs. The increases in BMD in loaded limbs were statistically significant $(p<0.001)$ in both the control and suspended animals (Fig. 3B). However, when the BMD of non-loaded limbs in the control and suspended groups was compared, no statistically significant differences were seen. The BMD of loaded limbs in each group was also not statistically different.

\section{Elevation in Akt-p and decrease in elF2a-p by knee loading}

Using Western blot analysis, the relative levels of Akt, Akt-p, elF2a, and elF2a-p in the femur and tibia were determined $1 \mathrm{~h}$ after loading on day 5 (Fig. 4). The level of Akt-p was shown to be greater in animals subjected to loading in both the control and suspended groups. In addition, the level of elF2a-p was lower in control and suspended animals. While the same trend was seen for both the femur and tibia, the response was stronger in the femur.

\section{Discussion}

The data herein demonstrated that knee loading significantly increased the BMD of hindlimb-suspended mice. While the effect was slightly stronger in the control mice, both groups showed a significant statistical difference $(p<0.001)$ from the non-loaded control limb. Previous experiments have shown the stimulatory effect of knee loading on bone formation in the hindlimbs of animals living in a normal, loaded environment (Zhang et al., 2006; Tanaka et al., 2004). Since the increase in BMD in each group was similar, data collected in this study suggest that unloaded limbs (hindlimb-suspended mice) respond in the same way as the loaded control (nonsuspended mice) to 5-day knee loading.

Western blot analysis revealed that phosphorylation of Akt increased in the presence of knee loading, while phosphorylation of elF2a was decreased. Because phosphorylation of Akt is a representative event of the activation of the PI3K pathway, data indicate that knee loading activates PI3K signaling. This result is consistent with previous results, in which PI3K was activated by mechanical loading (Zhang et al., 2009). The same trend was seen in both the hindlimb-suspended and control groups, indicating that unloading did not block the activation of the PI3k pathway. Note that the total Akt levels in hindlimb-suspended mice were reduced in the femur. A further analysis is needed to evaluate its contribution to a subsequent loss of BMD.

Unloading also did not affect the decrease in phosphorylation of elF2a, as both hindlimb-suspended and control mice exhibited a decrease in elF2a-p in response to knee loading. Since phosphorylation of elF2a has been shown to result from the application of ISR stress inducers, a decrease in its concentration may demonstrate a stress-reducing effect of knee loading. Less phosphorylated elF2a by knee loading indicates that translational initiation is inhibited to a lower degree and fewer cells are leading to apoptosis in both control and hindlimb-suspended mice. Between the femur and 
tibia, the effect of knee loading was stronger in the femur. This difference might be caused by their dependence on loading conditions and/or load distributions to the femur and tibia. A functional linkage of alterations in BMD in the femur and tibia to the phosphorylation levels of Akt and elF2a can be further investigated through BMD measurements and expression analysis at various locations throughout the femur and tibia.

The mean body weight of hindlimb-suspended mice was not statistically different from the mean body weight of non-suspended mice after day 5 of the study. In addition, there were no statistically significant differences in mean BMD of the entire body of the mouse between control and hindlimb-suspended mice. Although we did not directly measure an amount of food intake, mice appeared to eat and drink properly and were in a healthy condition. This result also demonstrates that the hindlimbsuspended mice did not experience a significant change in density of bone tissue throughout their body during the experiment. In the lumbar spine of the mouse, the mean BMD was slightly higher in hindlimb-suspended mice than in control mice; however, this difference was not statistically significant. The slight increase in BMD in hindlimb-suspended mice may be attributed to additional mechanical stress being applied to the lumbar section of the body, possibly increasing bone growth in that area.

This study demonstrated that knee loading, a form of mechanical stimulation, has a positive effect of bone formation in unloaded (hindlimb-suspended) mice. Other studies using mechanical stimulation on hindlimbsuspended mice have had similar results. Ozcivici et al., have reported that $90 \mathrm{~Hz}$ low-level whole-body vibrations applied for 15 minutes per day during the unloading period can help retain bone's ability to recover upon reamubulation. However, to our knowledge, this study is the first attempt to use knee loading to increase bone formation in unloaded mice.

Since the BMD of the entire body is not statistically different between control and hindlimb-suspended mice, 5 days of unloading may have only been enough to cause a minor case of disuse osteoporosis. A longer hindlimb-suspension study would likely cause a more severe case of this condition for knee loading to be tested on. In summary, we demonstrated that knee loading significantly increased bone formation in hindlimbsuspended mice. The results provide further evidence of the bone-producing capabilities of knee loading by demonstrating that this loading modality can be applied to unloaded limbs as well as limbs in normally-loaded environments. Individuals experiencing unloading, such as patients on bed rest or astronauts during space flight, are at a high risk of developing disuse osteoporosis. Therefore, knee loading may be a suitable mechanical treatment to strengthen bones and help prevent injuries related to unloading. Since 5-day hindlimb suspension did not induce significant reduction in BMD in the hindlimbs, a longer duration of hindlimb suspension should be employed to further examine efficacy of knee loading.

\section{Acknowledgements}

We appreciate Mina Tawfik for support in imaging. This study was supported by NASA NNX10AU51G, and NIH R01AR052144. The authors have no conflict of interest.

\section{References}

Akhter, M.P., Alvarez, G.K., Cullen, D.M., and Recker, R.R. (2011) Disuse-related decline in trabecular bone structure. Biomech. Model Mechanobiol., 10, 423-9.

Bergmann, P., Body, J.J., Boonen, S., Boutsen, Y., Devogelaer, J.P., Goemaere, S., Kaufman, J., Reginster, J.Y., and Rozenberg, S. (2010) Loading and skeletal development and maintenance. J. Osteoporos., 2011, 786752.

Cantley, L.C. (2002) The phosphoinositide 3-kinase pathway. Science., 296, 1655-7.

Hirasawa, H., Jiang, C., Zhang, P., Yang, F.C. and Yokota, H. (2010) Mechanical stimulation suppresses phosphorylation of elF2a and PERK-mediated responses to stress to the endoplasmic reticulum. FEBS Lett., 584, 745-752.

Iwamoto, J., Takeda, T., and Sato, Y. (2005) Interventions to prevent bone loss in astronauts during space flight. Keio J. Med., 54(2), 55-9.

Kwon, R., Meays, D., Tang, W., and Frangos, J. (2010) Microfluidic enhancement of intramedullary pressure increases interstitial fluid flow and inhibits bone loss in hindlimb suspended mice. J. Bone Miner. Res., 8, 1798-807.

Morey-Holton, E. and Globus, R.K. (1998) Hindlimb unloading of growing rats: a model for predicting skeletal changes during space flight. Bone, 5 Suppl, 83S-88S.

Ozcivici, E., Luu, Y.K., Rubin, C.T., and Judex, S. (2010) Low-level vibrations retain bone marrow's osteogenic potential and augment recovery of trabecular bone during reambulation. PLoS One., 5, e11178.

Proud, C.G. (2005) elF2 and the control of cell physiology. Semin. Cell Dev. Biol., 16, 3-12.

Qin, Y.X. and Lam, H. Intramedullary pressure and matrix strain induced by oscillatory skeletal muscle stimulation and its potential in adaptation. (2009) J. Biomech., 42(2), 140-5.

Ron, D. and Harding, H.P. (2007) elF2a phosphorylation in cellular stress responses and disease. Transl. Cont. Biol. Med., 13, 349-372.

Rubin, T., Gang, X., and Judex, S. (2001) The anabolic activity of bone tissue, suppressed by disuse, is normalized by brief exposure to extremely lowmagnitude mechanical stimuli. The FASEB J., 15, 2225-2229.

Sievänen, H. (2010) Immobilization and bone structure in humans. Arch. Biochem. Biophys., 503(1), 146-52. 
Tanaka, S., Sun, H., and Yokota, H. (2004) Bone Formation Induced by a Novel Form of Mechanical Loading on Joint Tissue. Biol. Sci. Space, 18, 41-44.

Zhang, P., Turner, C.H. and Yokota, H. (2009) Joint loading-driven bone formation and signaling pathways predicted from genome-wide expression profiles. Bone, 44, 989-998

Zhang, P., Su, M., Tanaka, S., Yokota, H. (2006) Knee loading stimulates cortical bone formation in murine femurs. BMC Musculoskelet Disord., 7, 73. 